\title{
openheart Dynamic cycling in atrial size and flow during obstructive apnoea
}

\author{
Gregg S Pressman, ${ }^{1}$ Beatriz Cepeda-Valery, ${ }^{1}$ Nicolas Codolosa, ${ }^{1}$ Marek Orban, ${ }^{2,3}$ \\ Solomon P Samuel, ${ }^{4}$ Virend K Somers ${ }^{5}$
}

To cite: Pressman GS, Cepeda-Valery B, Codolosa N, et al. Dynamic cycling in atrial size and flow during obstructive apnoea. Open Heart 2016;3:e000348. doi:10.1136/openhrt-2015000348

- Additional material is available. To view please visit the journal online (http://dx. doi.org/10.1136/openhrt2015-000348).

Received 1 October 2015 Revised 15 December 2015 Accepted 21 January 2016

\section{(a) CrossMark}

${ }^{1}$ Heart and Vascular Institute, Einstein Medical Center, Philadelphia, Pennsylvania, USA

${ }^{2}$ International Clinical Research Center, St Anne's University Hospital, Brno, Czech Republic

${ }^{3}$ Department of Human Pharmacology and Toxicology, Faculty of Pharmacy, University of Veterinary and Pharmaceutical Sciences, Brno, Czech Republic ${ }^{4}$ Office of Research and Technology Development, Einstein Medical Center, Philadelphia, Pennsylvania, USA

${ }^{5}$ Division of Cardiovascular Diseases, Mayo Clinic, Rochester, Minnesota, USA

Correspondence to Dr Gregg S Pressman; pressmang@einstein.edu

\section{ABSTRACT}

Objective: Obstructive sleep apnoea (OSA) is strongly associated with cardiovascular disease. However, acute cardiovascular effects of repetitive airway obstruction are poorly understood. While past research used a sustained Mueller manoeuver to simulate OSA we employed a series of gasping efforts to better simulate true obstructive apnoeas. This report describes acute changes in cardiac anatomy and flow related to sudden changes in intrathoracic pressure.

Methods and results: 26 healthy, normal weight participants performed 5-6 gasping efforts (target intrathoracic pressure $-40 \mathrm{~mm} \mathrm{Hg}$ ) while undergoing Doppler echocardiography. 14 participants had sufficient echocardiographic images to allow comparison of atrial areas during the manoeuver with baseline measurements. Mitral and tricuspid E-wave and A-wave velocities postmanoeuver were compared with baseline in all participants. Average atrial areas changed little during the manoeuver, but variance in both atrial areas was significantly greater than baseline. Further, an inverse relationship was noted with left atrial collapse and right atrial enlargement at onset of inspiratory effort. Significant inverse changes were noted in Doppler flow when comparing the first beat postmanoeuver (pMM1) with baseline. Mitral E-wave velocity increased $9.1 \mathrm{~cm} / \mathrm{s}$ while tricuspid E-wave velocity decreased $7.0 \mathrm{~cm} / \mathrm{s}$; by the eighth beat postmanoeuver ( $\mathrm{pMM} 8$ ) values were not different from baseline. Mitral and tricuspid A-wave velocities were not different from baseline at pMM1, but both were significantly higher by pMM8.

Conclusions: Repetitive obstructive apnoeas produce dynamic, inverse changes in atrial size and Doppler flow across the atrioventricular valves. These observations have important implications for understanding the pathophysiology of OSA.

Obstructive sleep apnoea (OSA) is highly prevalent in the general population, and is exacerbated by the current obesity epidemic. ${ }^{1}$ Various common cardiac diseases, such as atrial fibrillation and heart failure, major public health problems in their own right, are strongly linked to the presence of OSA. $^{2}$ The hallmarks of obstructive apnoea include hypoxaemia and the generation of high negative intrathoracic pressure. While

\section{KEY QUESTIONS}

What is already known about this subject?

- Obstructive sleep apnoea is strongly associated with atrial fibrillation but mechanisms linking the two remain unclear.

What does this study add?

- In response to a series of Mueller manoeuvers cyclic variations in left and right atrial size as well as flow through the mitral and tricuspid valves were observed.

How might this impact on clinical practice?

- Similar changes in atrial size and flow, repeated hundreds of times per night (in patients with obstructive sleep apnoea) might explain the frequent occurrence of atrial fibrillation in these patients.

much is known about the consequences of hypoxaemia, the acute cardiovascular effects of repetitive obstructive apnoeas are poorly understood. We undertook to use the Mueller manoeuver (forced inspiration against an occluded airway, MM), along with Doppler echocardiography, to better understand changes in cardiac anatomy and physiology that occur during obstructive apnoea.

Past research utilising the MM as a surrogate for OSA is limited by the fact that sustained negative inspiratory force was employed. This likely leads to a complex series of events with evolving changes in physiology from beginning to end of the manoeuver. ${ }^{3}$ By contrast, in actual obstructive apnoeas, repeated inspiratory efforts are made during each episode of airway obstruction. ${ }^{4}$ A novel feature of our current study is the use of repetitive gasping efforts to better simulate true obstructive apnoeas.

\section{METHODS}

Twenty-six male athletes (track and field) under age 30 were enrolled. All were healthy and on no medication. None was overweight or obese (body-mass index $<25$ in all 
participants) and all had good echocardiographic windows.

The MM was performed in the left lateral decubitus position. A nose clip and mouthpiece were positioned before each manoeuver; the mouthpiece incorporated a small air leak (via 3-way stopcock) to prevent complete closure of the glottis at the time of inspiratory effort. During the manoeuver, mouth pressure was visually monitored by participants, who were trained to generate a target intrathoracic pressure of $-40 \mathrm{~mm} \mathrm{Hg}(-54.4$ $\left.\mathrm{cm} \mathrm{H}_{2} \mathrm{O},-5.333 \mathrm{kPa}\right)$. After assuring that this goal was met, participants were coached in performing a series of five to six gasping efforts (as opposed to a sustained MM) over a period of $20 \pm 3 \mathrm{~s}$. Separate runs were made for atrial size and Doppler flow recordings. Between each run participants rested a few minutes to allow baseline physiology to be re-established.

For measurement of atrial area, two-dimensional echocardiographic imaging was performed from the apical four-chamber view. Baseline recordings were acquired during quiet respiration (5-8 cardiac cycles). Twenty second loops were then recorded taking care to assure that both atria were optimally visualised throughout. Recording started a few beats before onset of the manoeuver and continued during performance of the series of gasping efforts. These were repeated as needed to obtain adequate echocardiographic images.

For Doppler flow recordings pulsed wave Doppler flow imaging across the mitral and (separately) tricuspid valves was performed with the sample volume positioned between the leaflet tips when the valve was fully open. As before, participants rested a few minutes between runs to allow baseline physiology to be re-established. We attempted to record pulsed Doppler measurements during actual performance of the MM, but in most participants it was not possible to assure proper positioning of the sample volume while participants were making respiratory efforts. We therefore focused our attention on recordings made at baseline (during quiet respiration) and immediately on release of the MM, extending out at least eight cardiac cycles postmanoeuver.

Using the real-time two-dimensional images at baseline and during the MM, area of the right and left atria were traced for each beat using electronic calipers; values were recorded in systole when the atrium is largest. We also recorded, at baseline and during the MM, whether the mitral or tricuspid valve was the first to open in diastole, or whether both valves opened simultaneously. Using the Doppler flow recordings, mitral and tricuspid valve $\mathrm{E}$-wave and A-wave velocities were measured using electronic calipers, both at baseline and for each beat following completion of the MM.

All echocardiographic and Doppler measurements were made by a single reviewer blinded to the phase of the Mueller manoeuver.

This study was approved by the Institutional Review Board of the Albert Einstein Healthcare Network. All participants provided written informed consent.

\section{Statistical analysis}

The analysis of atrial area measurements proceeded as follows for the left atrium (LA) and right atrium (RA) separately. Each of the participants with atrial area measurements had the mean and variance of his baseline values calculated, and the mean and variance of his MM values calculated. Student's unpaired t test compared the grand average, using the pooled variance, at baseline to that during the MM, separately for the LA and RA, at a two-sided significance level of 0.05 without multiplicity correction. Fisher's F-test compared the variation in atrial area during the MM to that at baseline for each atrium separately using a 0.05 significance level.

The analysis of Doppler velocity measurements proceeded as follows, separately, for the E-wave and for the A-wave of each valve (mitral and tricuspid). For each of the 26 participants with velocity measurements, the average of his baseline values was compared in pairwise fashion to the value at his first cardiac cycle post-MM (pMM1) and separately, to that of his eighth cardiac cycle post-MM (pMM8). The pMM1 beat most closely represents that during the MM and the pMM8 beat represents short-term recovery from the MM. Paired Student's $t$ test performed these comparisons at a twosided significance level of 0.05 without multiplicity correction.

\section{RESULTS}

Baseline echocardiographic and Doppler variables are shown in table 1 .

\section{Chamber area measurements during the Mueller manoeuver}

A total of 14 participants had echocardiographic imaging throughout the MM that was of sufficient quality for analysis. A striking finding was the immediate 'collapse' of the LA with inspiratory effort which reversed as soon as the negative inspiratory force was released (figure 1 and online supplementary video 1 ). In addition this pattern of sudden collapse followed by sudden expansion of the LA occurred with each inspiratory effort. The RA moved in inverse fashion to the left (figure 2).

\begin{tabular}{|c|c|}
\hline Septal wall thickness $(\mathrm{cm})$ & $0.8 \pm 0.1$ \\
\hline Posterior wall thickness $(\mathrm{cm})$ & $0.8 \pm 0.1$ \\
\hline LV dimension (end-diastole, $\mathrm{cm}$ ) & $4.2 \pm 0.5$ \\
\hline LA area $\left(\mathrm{cm}^{2}\right)$ & $17.0 \pm 2.3$ \\
\hline $\mathrm{RA}$ area $\left(\mathrm{cm}^{2}\right)$ & $16.7 \pm 3.1$ \\
\hline Mitral E-wave velocity (cm/s) & $83.7 \pm 16.4$ \\
\hline Mitral A-wave velocity (cm/s) & $38.8 \pm 9.2$ \\
\hline Tricuspid E-wave velocity (cm/s) & $54.1 \pm 7.7$ \\
\hline Tricuspid A-wave velocity (cm/s) & $26.3 \pm 6.4$ \\
\hline
\end{tabular}


Figure 1 Still frames from the apical four-chamber view showing dynamic change in left atrial size.

The image on the left was captured with the onset of inspiratory effort and shows the sudden 'collapse' of the left atrium. The image on the right was captured at release of the Mueller manoeuver (MM) and shows a much larger left atrium. Note the reciprocal changes in right atrial size.
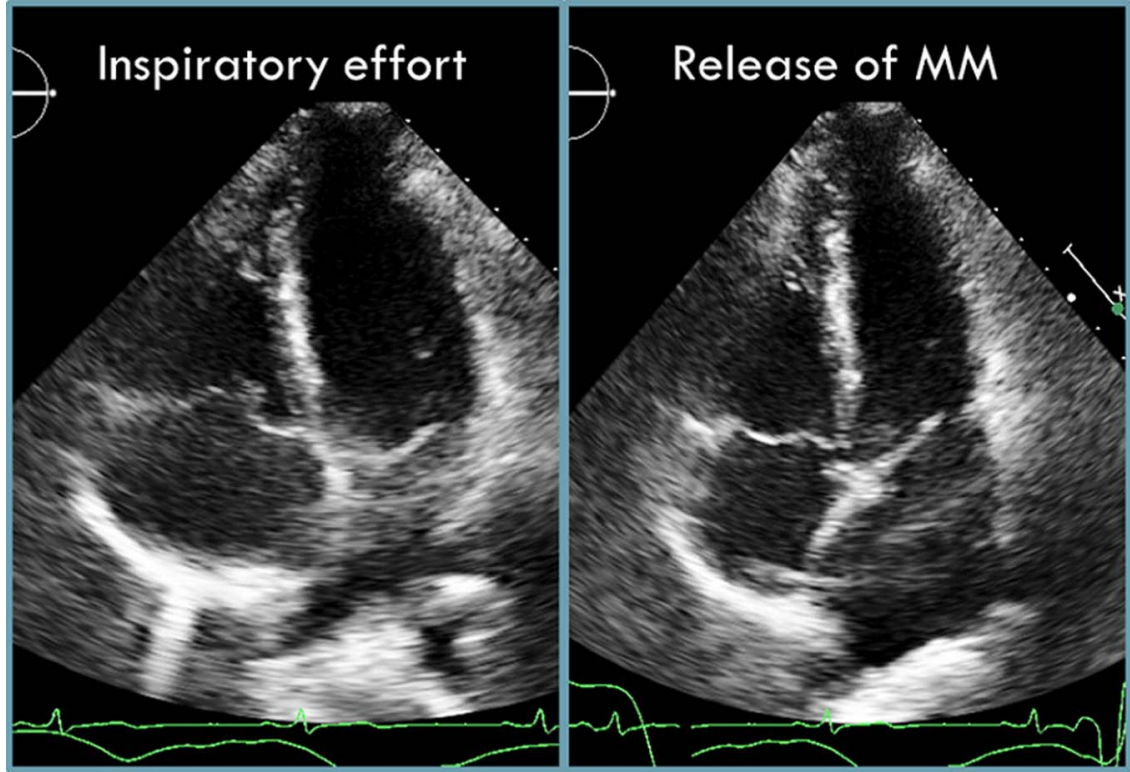

These 14 participants contributed a median of 8 (range 4-9) baseline and 18 (range 11-24) MM left atrial, and 8 (range 4-9) baseline and 17 (range 9-25) $\mathrm{MM}$ right atrial measurements. Mean baseline left atrial area (measured at end-systole, $\mathrm{N}=99$ beats) was 16.6 $\pm 0.84 \mathrm{~cm}^{2}$ (mean \pm pooled SD); mean baseline right atrial area ( $\mathrm{N}=100$ beats) was $16.3 \pm 0.93 \mathrm{~cm}^{2}$. During the MM mean LA area ( $\mathrm{N}=252$ beats) was $16.7 \pm 1.29 \mathrm{~cm}^{2} \mathrm{p}=\mathrm{NS}$ versus baseline) while mean $\mathrm{RA}$ area ( $\mathrm{N}=253$ beats) was $15.4 \pm 1.69 \mathrm{~cm}^{2} \quad(p=0.02$ vs baseline). For left and right separately, variance in atrial area during the MM significantly exceeded that at baseline $(p<0.0001$ for each, F-test). During the MM, LA variance increased to 2.38-fold that of baseline while RA variance increased to 3.28-fold that of baseline.

\section{Doppler variables during the Mueller manoeuver}

Doppler data at baseline and immediately following the series of gasping efforts were available in all 26 participants (table 2 and figure 3 ).

As noted above, there were too few participants with adequate Doppler recordings during the MM to allow meaningful analysis. These 26 participants contributed a median of 10 (range 6-13) mitral E-wave, 10 (range 615) mitral A-wave, 11 (range 8-13) tricuspid E-wave, and 11 (range 8-13) tricuspid A-wave baseline measurements. For all participants the mitral valve average E-wave velocity at baseline was $83.66 \pm 16.38 \mathrm{~cm} / \mathrm{s}$ and average A-wave velocity was $38.78 \pm 9.17 \mathrm{~cm} / \mathrm{s}$. At pMM1 the average E-wave velocity was increased by $9.1 \mathrm{~cm} / \mathrm{s}$ $(p=0.02)$. At $p M M 8$ average E-wave velocity had returned towards baseline but was still $4.5 \mathrm{~cm} / \mathrm{s}$ higher $(\mathrm{p}=0.058$ vs baseline). By contrast average A-wave velocity at pMM1 was not significantly different from baseline. By pMM8 average A-wave velocity increased by $9.8 \mathrm{~cm} / \mathrm{s}$ $(\mathrm{p}<0.0001)$.
Regarding the tricuspid valve, average E-wave velocity at baseline was $54.11 \pm 7.73 \mathrm{~cm} / \mathrm{s}$ and average A-wave velocity was $26.32 \pm 6.44 \mathrm{~cm} / \mathrm{s}$. At pMM1 average E-wave velocity decreased by $7.0 \mathrm{~cm} / \mathrm{s}(\mathrm{p}=0.001)$. At pMM8 average E-wave velocity had returned to baseline. Average A-wave velocity behaved similarly to that of the mitral valve, with no significant change at pMM1 but a significant increase of $4.5 \mathrm{~cm} / \mathrm{s}$ at pMM8 $(\mathrm{p}=0.04)$.

\section{Valve opening}

At baseline, in all participants, opening of the mitral and tricuspid valves occurred simultaneously or the tricuspid valve opened first. There was little or no variation over time in valve opening pattern for any individual participant at rest. By contrast, during performance of the MM there was marked variation in valve opening pattern within participants, corresponding to the series of gasping efforts performed. Figure 4 shows one example in which both valves open simultaneously at rest. However, during the MM there is cyclic alternation between simultaneous valve opening and the tricuspid valve opening first. This type of rhythmic variation in valve opening pattern occurred in every participant during the MM.

\section{DISCUSSION}

In this study we documented dynamic cycling in atrial size and flow related to repetitive inspiratory efforts. This is a new finding which may have implications for understanding the strong association between OSA and atrial fibrillation. Previous research employing the MM as a surrogate for naturally occurring obstructive apnoea utilised a sustained inspiratory effort. Our aim was to more closely mimic OSA through a series of gasping efforts against a closed airway. The main findings relate to induction of a hyperdynamic state with phasic 

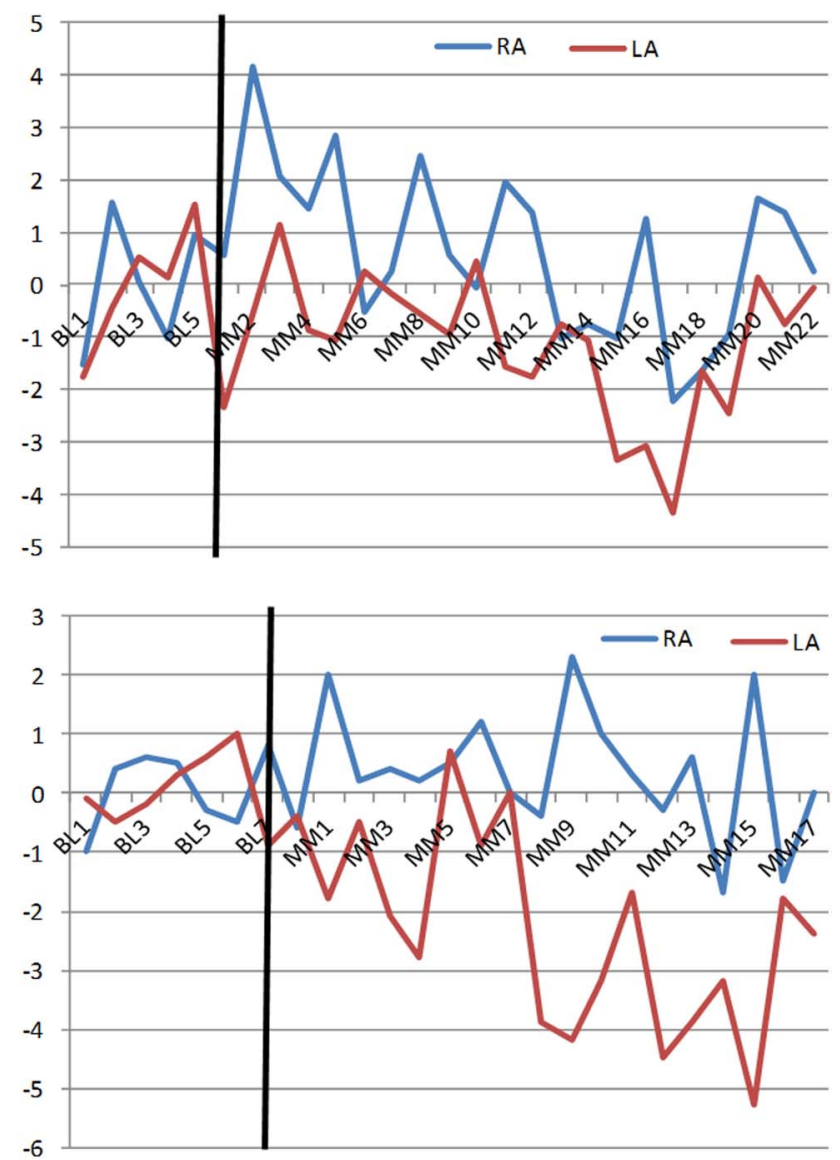

Figure 2 Representative graphs for two participants illustrating the inverse variation in left and right atrial areas during the series of gasping efforts. The vertical black line indicates onset of the Mueller manoeuver. Heart beats are counted on the $\mathrm{X}$-axis $(\mathrm{BL}=$ baseline, $\mathrm{MM}=$ Mueller manoeuver). The $\mathrm{Y}$-axis indicates deviation in atrial area from the baseline average (in $\mathrm{cm}$ ), on a beat-to-beat basis. With each inspiratory effort the left atrium (LA) becomes smaller while the right atrium (RA) enlarges. These changes reverse when the negative intrathoracic pressure is relieved. In each of these cases it can be appreciated that the participant made five inspiratory efforts.

changes noted in both atrial size and in Doppler flows through the mitral and tricuspid valves. The most striking observation was that of left atrial collapse occurring immediately with onset of negative inspiratory pressure. There was also a reciprocal relationship between left and right atrial areas which moved in phase with the gasping efforts. Similarly, there was a reciprocal relationship in Doppler flow velocity across the atrioventricular valves: an immediate and significant increase in the mitral E-wave velocity occurred on release of the manoeuver, with a concomitant decrease in tricuspid E-wave velocity. These findings imply increased flow across the mitral valve at the same time tricuspid valve flow decreases. The observed changes reversed by eight beats after offset of the MM. In support of dynamic changes in the circulation we also found marked variation in the pattern of valve opening during the series of gasping efforts.
Table 2 Change in Doppler variables from baseline to post-Mueller manoeuver

\begin{tabular}{lll}
\hline Variable & $\begin{array}{l}\text { Change from } \\
\text { baseline to } \\
\text { pMM1 }\end{array}$ & $\begin{array}{l}\text { Change from } \\
\text { baseline to } \\
\text { pMM8 }\end{array}$ \\
\hline $\begin{array}{l}\text { Mitral E-wave } \\
\text { velocity (cm/s) }\end{array}$ & $+9.14, p=0.02$ & $+4.45, p=0.06$ \\
$\begin{array}{l}\text { Tricuspid E- } \\
\text { wave velocity } \\
\text { (cm/s) }\end{array}$ & $-\mathbf{7 . 0 4 , p = 0 . 0 0 0 6}$ & $+0.14, p=0.94$ \\
$\begin{array}{l}\text { Mitral A-wave } \\
\text { velocity (cm/s) }\end{array}$ & $+3.03, p=0.13$ & $+9.84, p<0.0001$ \\
$\begin{array}{l}\text { Tricuspid A- } \\
\text { wave velocity } \\
\text { (cm/s) }\end{array}$ & $+0.43, p=0.79$ & $+\mathbf{4 . 5 0 , p = 0 . 0 4}$ \\
\hline $\begin{array}{l}\text { pMM1=first beat after release of the Mueller manoeuver; } \\
\text { pMM8=eighth beat after release of the Mueller } 0 ; p \text { value is for } \\
\text { comparison with pMM1 or pMM8 with baseline; significant values } \\
\text { in bold. }\end{array}$
\end{tabular}

The collapse of the LA with onset of negative inspiratory force is striking and requires explanation. Previous work has documented increased flow into the right heart with onset of negative pressure in the chest. ${ }^{5-7}$ It is also known that performance of a MM imposes an afterload burden on the left ventricle, resulting in reduced stroke volume and an increase in end-systolic volume. ${ }^{8-}$ 10 These changes occur suddenly and in a constrained pericardial space; together they might leave little room for the LA resulting in the sudden decrement in left atrial size. Flow into the left ventricle is hampered by the

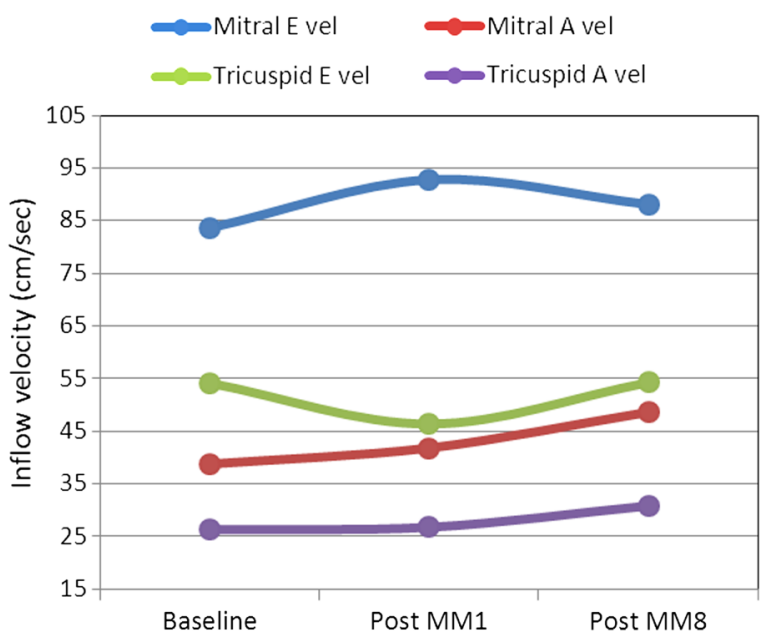

Figure 3 Doppler E-wave and A-wave velocities across the mitral and tricuspid valves at different time points. Immediately on release of the Mueller manoeuver (post-MM1) there is a significant rise in mitral E-wave velocity and an inverse fall in tricuspid E-wave velocity as compared with baseline. By 8 beats after release (post-MM8) these values have returned to baseline. By contrast the mitral and tricuspid A-wave velocities at release are not significantly different from baseline. Eight beats later, though, they are both increased significantly. 


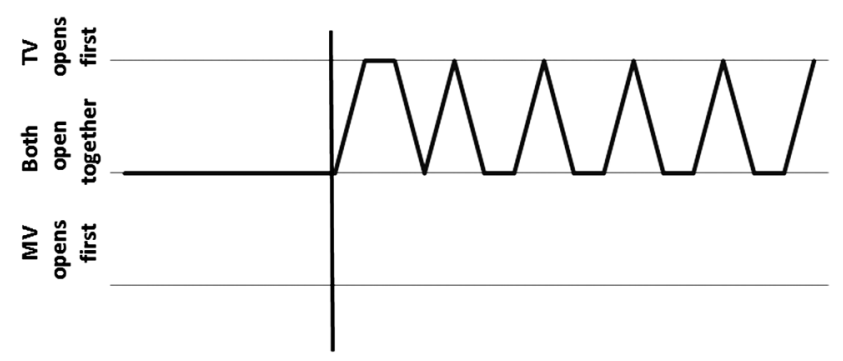

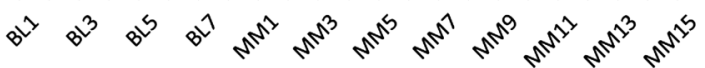

Figure 4 Representative graph of valve opening for a single participant. The vertical black line indicates onset of the Mueller manoeuver. Heart beats are counted on the X-axis ( $B L=$ baseline, $M M=M u e l l e r$ manoeuver); the $y$-axis indicates whether the tricuspid valve (TV) opens first in diastole or the mitral valve (MV) or both simultaneously. In this example, it can be appreciated that both valves open together at baseline. By contrast, the series of gasping efforts produces a new opening pattern where the tricuspid valve opens first during each of the inspiratory efforts.

increased afterload generated by the MM but negative intrathoracic pressure should allow pooling of blood in the pulmonary vasculature. Thus, it is conceivable that the left atrial collapse is associated with reversal of flow in the pulmonary veins. In fact, normal pulmonary venous flow into the LA results from a combination of a 'suction' event due to descent of the mitral annulus in systole (lessened during the MM), and propagation of a forward-going pressure wave from the right ventricle (likely reduced or reversed during the MM due to pooling in the pulmonary bed). ${ }^{11}$ Proof of this explanation would require documentation of reverse flow in the pulmonary veins during the MM. Future study with invasive haemodynamics would be very revealing in this regard.

\section{Clinical importance}

OSA is strongly associated with atrial fibrillation, both in experimental models ${ }^{12}$ and clinical studies. ${ }^{13-15}$ However, specific pathophysiological mechanisms are still being worked out. Atrial stretch is known to contribute to the genesis and maintenance of atrial fibrillation $^{16}$ and OSA is associated with enlargement of the LA. ${ }^{17}$ Atrial natriuretic peptide levels have also been found to be increased in patients with OSA and to decrease with treatment. ${ }^{18}$ Based on the current findings, there appear to be dynamic changes in atrial size and flow across the atrioventricular valves that occur in concert with swings in intrathoracic pressure. These changes could affect the electrophysiological properties of the LA and adjacent pulmonary vein ostia, the site of electrical triggers that often initiate atrial fibrillation. Given that obstructive apnoeas occur dozens or even hundreds of times per night, it is conceivable that these changes ultimately result in atrial enlargement and predispose to atrial tachyarrhythmias.

\section{Limitations}

We employed the MM as a surrogate for naturally occurring apnoeas. Unlike the case in true OSA, the MM alone does not elicit significant hypoxaemia, and participants are awake so the effects of the arousals that occur with OSA are not incorporated into the changes we describe. However, use of the MM allows us to focus specifically on the haemodynamic effects of negative intrathoracic pressure per se without introducing the complicating influences of these other factors. Further, like true OSA, the MM produces elevations in blood pressure and left ventricular afterload. Finally, as noted above, through use of a series of gasping efforts, we were able to overcome a limitation of the traditional MM and more closely simulate what happens in naturally occurring OSA.

Participants were studied in the left lateral decubitus position. This was necessary in order to optimise echocardiographic imaging. However, patients with OSA often sleep on their backs. It is possible that the changes observed in this study would be different if participants had laid supine.

We studied only men but there is no reason to expect that acute changes in cardiovascular physiology with application of negative intrathoracic pressure would differ between men and women.

Last, our sample size was modest. In mitigation, however, the findings were striking and reproducible. We were also able to document phasic changes in atrial physiology by three different means: inverse changes in left and right atrial size, reciprocal changes in mitral and tricuspid E-wave velocities and changes in the pattern of mitral and tricuspid valve opening in diastole.

\section{CONCLUSIONS}

Sudden changes in intrathoracic pressure, the hallmark of obstructive apnoea, elicit dynamic changes in atrial size and flow across the atrioventricular valves. Within the constrained pericardial space, size of the left and right atria vary inversely and in concert with inspiratory effort. Similarly, flow across the mitral and tricuspid valves changes dramatically and inversely, again according to changes in intrathoracic pressure. These observations have important implications for understanding the mechanisms underlying the strong association between OSA and atrial fibrillation.

Contributors BC-V, NC, MO, and SPS contributed to the study design and data analysis. GSP and VKS are responsible for interpretation of results and the writing of the manuscript.

Funding This research was supported by a grant from the Pennsylvania Department of Health and by The Women's League for Medical Research, Einstein Medical Center, Philadelphia, PA, USA.

Competing interests VKS reports that the Mayo Foundation has received a gift from the Philips Respironics Foundation for the study of sleep and 
cardiovascular disease. He has served as a consultant to U-Health, GlaxoSmithKline, Price Waterhouse Coopers, Rhonda Grey, ResMed, Philips, Sorin Inc., and is working with Mayo Health Solutions and their industry partners on intellectual property related to sleep and cardiovascular disease

Ethics approval Albert Einstein Healthcare Network IRB.

Provenance and peer review Not commissioned; externally peer reviewed.

Open Access This is an Open Access article distributed in accordance with the Creative Commons Attribution Non Commercial (CC BY-NC 4.0) license, which permits others to distribute, remix, adapt, build upon this work noncommercially, and license their derivative works on different terms, provided the original work is properly cited and the use is non-commercial. See: http:// creativecommons.org/licenses/by-nc/4.0/

\section{REFERENCES}

1. Drager LF, Togeiro SM, Polotsky VY, et al. Obstructive sleep apnea: a cardiometabolic risk in obesity and the metabolic syndrome. J Am Coll Cardiol 2013;62:569-76.

2. Somers VK, White DP, Amin R, et al. Sleep apnea and cardiovascular disease: an American Heart Association/American College of Cardiology Foundation Scientific Statement from the American Heart Association Council for High Blood Pressure Research Professional Education Committee, Council on Clinical Cardiology, Stroke Council, and Council On Cardiovascular Nursing. In collaboration with the National Heart, Lung, and Blood Institute National Center on Sleep Disorders Research (National Institutes of Health). Circulation 2008;118:1080-111

3. Hall MJ, Ando S, Floras JS, et al. Magnitude and time course of hemodynamic responses to Mueller maneuvers in patients with congestive heart failure. J Appl Physiol 1998;85:1476-84.

4. Deegan PC, McNicholas WT. Pathophysiology of obstructive sleep apnoea. Eur Respir J 1995;8:1161-78.

5. Santamore WP, Heckman JL, Bove AA. Right and left ventricular pressure-volume response to respiratory maneuvers. J App Physiol Respir Environ Exerc Physiol 1984;57:1520-7.

6. Guzman PA, Maughan WL, Yin FC, et al. Transseptal pressure gradient with leftward septal displacement during the Mueller manoeuvre in man. Br Heart $J$ 1981;46:657-62.
7. Condos WR Jr, Latham RD, Hoadley SD, et al. Hemodynamics of the Mueller maneuver in man: right and left heart micromanometry and Doppler echocardiography. Circulation 1987;76: 1020-8.

8. Buda AJ, Pinsky MR, Ingels NB Jr, et al. Effect of intrathoracic pressure on left ventricular performance. $N$ Engl J Med 1979;301:453-9.

9. Orban M, Bruce CJ, Pressman GS, et al. Dynamic changes of left ventricular performance and left atrial volume induced by the mueller maneuver in healthy young adults and implications for obstructive sleep apnea, atrial fibrillation, and heart failure. Am J Cardiol 2008;102:1557-61.

10. Koshino $Y$, Villarraga HR, Orban $M$, et al. Changes in left and right ventricular mechanics during the Mueller maneuver in healthy adults: a possible mechanism for abnormal cardiac function in patients with obstructive sleep apnea. Circ Cardiovasc Imaging 2010;3:282-9.

11. Smiseth OA, Thompson CR, Lohavanichbutr $\mathrm{K}$, et al. The pulmonary venous systolic flow pulse--its origin and relationship to left atrial pressure. J Am Coll Cardiol 1999;34:802-9.

12. Iwasaki $\mathrm{YK}$, Kato $\mathrm{T}$, Xiong $\mathrm{F}$, et al. Atrial fibrillation promotion with long-term repetitive obstructive sleep apnea in a rat model. $J \mathrm{Am}$ Coll Cardiol 2014;64:2013-23.

13. Gami AS, Pressman G, Caples SM, et al. Association of atrial fibrillation and obstructive sleep apnea. Circulation 2004; 110:364-7.

14. Kanagala R, Murali NS, Friedman PA, et al. Obstructive sleep apnea and the recurrence of atrial fibrillation. Circulation 2003;107:2589-94.

15. Gami AS, Hodge DO, Herges RM, et al. Obstructive sleep apnea, obesity, and the risk of incident atrial fibrillation. J Am Coll Cardiol 2007;49:565-71.

16. De Jong AM, Maass AH, Oberdorf-Maass SU, et al. Mechanisms of atrial structural changes caused by stretch occurring before and during early atrial fibrillation. Cardiovas Res 2011;89:754-65.

17. Oliveira W, Campos O, Bezerra Lira-Filho E, et al. Left atrial volume and function in patients with obstructive sleep apnea assessed by real-time three-dimensional echocardiography. J Am Soc Echocardiogr 2008;21:1355-61.

18. Svatikova A, Shamsuzzaman AS, Wolk R, et al. Plasma brain natriuretic peptide in obstructive sleep apnea. Am J Cardio 2004:94:529-32. 